\title{
Foam Mat Drying Characteristics of Mango Pulp
}

\author{
Robin A. Wilson, Dattatre ya M. Kadam*, Sonia Chadha , Monika Sharma \\ Central Institute of Post-Harvest Engineering and Technology (CIPHET), Ludhiana, Punjab, India
}

\begin{abstract}
Mango is widely preferred because of its excellent flavour and nutritional quality. Mango pulp from Dussehri variety was foam mat dried using $0,3,5,7$, and $9 \%$ egg white as foaming agent and then dried at air dry ing temperature of 65 , 75 and $85^{\circ} \mathrm{C}$. Weight loss was used to estimate change in mo iture ratio with respect to time and effective diffusivity. Seven thin layer drying models were fitted to get the best fit model, which was selected on the bas is of various statistical para meters. Wang and Singh model was found to be best in almost all cases. Nutritional status in terms of total carotenes was estimated and it was observed that there was significant effect of drying temperatures and egg white concentration. Based on above parametres it was resolved that foam mat drying using $3 \%$ egg white at $65^{\circ} \mathrm{C}$ air dry ing temperature was the best combination.
\end{abstract}

Keywo rds Mango, Foam Mat Drying, Egg White, Dry ing Kinetics, Effective Moisture Diffusivity, Carotenes

\section{Introduction}

Mango (Mangifera indica L.) commonly known as king of fruits is a major fruit in Asia and around the world. Nutritional importance of mango is mainly due to carotenes and other bioactive compounds[1]. India being the largest producer of mango contributes $37 \%$ of total 30.5 million tons of global production[2]. Mango impose greater problem in storage and transportation, as it is highly perishable than other tropical and sub tropical fru its. Wide gap between total production and consumption due to poor transportation and storage facilities leads to post harvest losses[3]. To avoid post harvest losses and increase the shelf life mango has to be processed into shelf stable products. Conventional types of mango products have been developed to a considerable extent but the mango industry is eager to develop new processed products[4]. In India, few studies have been reported on the development of mango powder. Spray drying of mango pulp produced good coloured powder but no pleasant flavour[5]. Freeze-dried mango pulp with added sugar produced powder with good shelf life but the cost was prohibitive[6]. Sweetened mango powder produced by drying, which involved mixing pulp with an equal quantity of sucrose and dried in a vacuum drier at $65^{\circ} \mathrm{C}$ and $27^{\prime \prime} \mathrm{Hg}$ vacuum for $8 \mathrm{~h}$, retained $57 \% \beta$-carotene at $38^{\circ} \mathrm{C}$ and $65 \%$ $\mathrm{RH}[7]$.

Foaming of liquid and semi-liquid materials has long been recognized as one of the efficient methods to shorten drying time. Over the past decade, this relatively old technology, known as foam mat drying, received renewed attention

* Corresponding author:

kadam1k@yahoo.com (Dattatreya M. Kadam)

Published online at http://journal.sapub.org/food

Copyright (C) 2012 Scientific \& Academic Publishing. All Rights Reserved because of its added ability to process hard-to-dry materials to produce products of desired properties, retaining volatiles that otherwise would be lost during the drying of non-foamed materials[8,9]. Foam mat drying involves the incorporation of foaming agent into liquid foods with subsequent whipping to form stiff foam[10,11]. In general, drying rate of foamed materials is faster than non-foamed materials and is greatly accelerated during final stages of drying. Many researchers have reported that the increased interfacial area of foamed materials is the factor responsible for its enhanced drying rate.

Present investigation was aimed to study the foam mat drying characteristics of mango pulp to enhance its shelf life and finding optimal conditions to apply this technique for mango powder development with retention of nutritional quality.

\section{Material and Methods}

\subsection{Drying Experiment}

Fully ripened mangoes (Dushehri variety) were purchase $\mathrm{d}$ from the local market of Ludhiana (Punjab). Mangoes were washed with water to remove dirt and foreign matter, if any. After manual grading for uniform size and shape, only sound, blemish free fruits were taken for extracting pulp. Pulp was homogenized using domestic mixer after peeling of skin and stone removal. Sample size of $300 \mathrm{~g}$ in triplicate was agitated using hand blender (Orpat-HHB100E, Ajanta Limited, India.) at 18000rpm. Egg white (EW) @ 0, 3, 5, 7 and $9 \%$ was added to develop the foam which increases surface area due to air incorporation. Foa med pulp was spread in food grade stainless steel trays and dried in tray dryer (MSW-210, Macro Scientific Work, India) at three different drying air temperature of 65,75 and $850 \mathrm{C}$. 
Weight loss was measured after every half an hour to determine drying rate and other drying parameters. The semi dried foam mats were peeled at around 15-19\% mo isture content for faster drying and better quality retention. Peeled mats were reversed and dried for another half an hour in order to reduce mo is ture content of the mats below $3 \%$. The dried mats were pulverized and packing for further studies.

\begin{tabular}{cc} 
& Nomenclature \\
\hline Abbreviation & Full form \\
\hline$\chi^{2}$ & Reduced chi-square \\
$\mathrm{a}, \mathrm{b}, \mathrm{c}, \mathrm{n}$ & Empirical constants in drying models \\
$\mathrm{D}_{\text {eff }}$ & Effect ive moisture diffusivity, $\mathrm{m}^{2} / \mathrm{s}$ \\
$\mathrm{K}$ & Drying constant \\
$\mathrm{L}$ & Thickness of foam mat, $\mathrm{m}$ \\
$\mathrm{M}$ & Moisture content at timet, $\mathrm{kg}$ moist ure. \\
$\mathrm{M}_{\mathrm{e}}$ & Equilibrium moist ure content, $\mathrm{kg}$ moist ure. \\
$\mathrm{M}_{\mathrm{o}}$ & Initial moisture content, $\mathrm{kg}$ moist ure. \\
$\mathrm{MR}$ & Dimensionless moisture ratio \\
$\mathrm{N}$ & Number of observations \\
$\mathrm{R}^{2}$ & Coefficient of determination \\
$\mathrm{RMSE}$ & Root mean square error \\
$\mathrm{MBE}$ & Mean biased error \\
$\mathrm{T}$ & Drying time, $\mathrm{h}$ \\
$\mathrm{Z}$ & Number of drying constants \\
$\mathrm{EW}$ & Egg white \\
\hline
\end{tabular}

\subsection{Moisture Ratio}

Moisture ratio of samples during drying was determined using following equation:

$$
\mathrm{MR}=\left(\mathrm{M}-\mathrm{M}_{\mathrm{e}}\right) /\left(\mathrm{M}_{\mathrm{o}}-\mathrm{M}_{\mathrm{e}}\right)
$$

As the $M_{e}$ value is very small compared to $M_{o}$ and $M$ values, the $M_{e}$ value can be neglected and the mo isture ratio was simplified and it can be expressed as $[9,12]$.

$$
\mathrm{MR}=\mathrm{M} / \mathrm{M}_{\mathrm{o}}
$$

\subsection{Moisture Diffusi vity}

Fick's diffusion equation for particles with slab geometry was used for calculation of effective mo isture diffusivity. Thin layered foamed mango pulp in a tray was considered as slab geometry[13]. The equation is expressed as[14]:

$$
M R=\frac{8}{\pi^{2}} \exp \left(\frac{-\pi^{2} D_{e f f} t}{4 L^{2}}\right)
$$

Equation (3) can be rewritten as:

$$
D_{\text {eff }}=\frac{\ln M R-\ln \frac{8}{\pi^{2}}}{\left(\frac{\pi^{2} t}{4 L^{2}}\right)}
$$

The slope $\left(\mathrm{K}_{\mathrm{o}}\right)$ is calculated by plotting $\mathrm{Ln}(\mathrm{MR})$ vs. time according to equation (6) to determine the effective diffusivity for different temperatures.

$$
k_{o}=\left(\frac{\pi^{2} D_{e f f}}{4 L^{2}}\right)
$$

To select a suitable model for describing the foam mat drying process of mango, drying curves were fitted with seven thin layer drying equations. The evaluated moisture ratio models are presented in Table 1 . The non-linear regression analysis was done using STATISTICA 6.0 (Stat soft). Coefficient of determination, $R^{2}$ was one of the main criteria for selecting the best model. In addition to $R^{2}$, the goodness of fit was determined by various statistical parameters such as reduced chi-square $\left(\chi^{2}\right)$, Root mean square error (RMSE) and Mean bias error (MBE). For quality fit, $R^{2}$ value should be higher and $\chi^{2}, \mathrm{MBE}$ and RMSE values should be lower[9, 12, 15].

Table 1. Thin Layer Drying Models and Their Equations

\begin{tabular}{cc}
\hline Model & Equation \\
\hline Newton [22] & $\mathrm{MR}=\operatorname{Exp}(-\mathrm{kt})$ \\
Henderson and Pabis[23,24] & $\mathrm{MR}=\mathrm{a} \operatorname{Exp}(-\mathrm{kt})$ \\
Logarithmic[25] & $\mathrm{MR}=\mathrm{a} \operatorname{Exp}(-\mathrm{kt})+\mathrm{c}$ \\
Two term[26, 27] & $\mathrm{MR}=\mathrm{a} \operatorname{Exp}(-\mathrm{kt})+\mathrm{b} \operatorname{Exp}(-\mathrm{nt})$ \\
Twoterm exponential[28] & $\mathrm{MR}=\mathrm{a} \operatorname{Exp}(-\mathrm{kt})+(1-\mathrm{a}) \operatorname{Exp}(-\mathrm{kat})$ \\
Wang and Singh[29] & $\mathrm{MR}=1+(\mathrm{at})+\left(\mathrm{bt}{ }^{2}\right)$ \\
Diffusion approach[30] & $\mathrm{MR}=\mathrm{a} \operatorname{Exp}(-\mathrm{kt})+(1-\mathrm{a}) \operatorname{Exp}(-\mathrm{kbt})$ \\
\hline
\end{tabular}

\subsection{Nutritional Quality}

Nutritional quality in terms of total carotene content was estimated using the Rangana[16] method.

\section{Results and Discussion}

\subsection{Drying Characteristics}

Moisture content of fresh mangoes was found to be 79.25 $\pm 1.77 \%$ (wet basis). The average drying time for foam mat drying was 390,330 and $300 \mathrm{~min}$ at 65,75 and $85^{\circ} \mathrm{C}$ respectively (Figure 1). The final moisture content of powdered samples was in the range of $1.12-3.05 \%$. Drying air temperature had significant effect on drying time, which is evident from the fact that drying time, reduced with increase in drying air temperature. Mo isture reduction per hour was higher at initial stages and then started to decrease with the increasing drying time. The results are in accordance with the earlier observations for foam mat drying of tomato[9]. It was observed that drying occurred primarily in falling rate period and no constant rate period was observed at all drying temperatures. It can be deduced from Figure1 that concentration of foaming agent has significant effect on drying rate. At $65^{\circ} \mathrm{C}$ drying, sample with $3 \%$ egg white had minimum mo isture ratio with respect to time, however in case of drying at 75 and $85^{\circ} \mathrm{C}$, minimum mo isture ratio was noticed with 3 and $5 \%$ and 3 and $7 \%$ egg white respectively. From above it can be concluded that $3 \%$ egg white concentration is good due to effective drying rate. Although samples with 5 and $7 \%$ egg white had similar drying rate, but being higher concentrations, these could be

\subsection{Model Fitting}


avoided to prevent excessive use of foaming agent.


Figure 1. Effect of egg white (\%) on drying rate of foam mat dried mango pulp at (A) $65^{\circ} \mathrm{C}$, (B) $75^{\circ} \mathrm{C}$ and (C) $85^{\circ} \mathrm{C}$ drying air temperat ures

\subsection{Moisture Diffusi vity}

The effective moisture diffusivity ranged between $1.53 \times 10^{-8}$ and $2.63 \times 10^{-8} \mathrm{~m}^{2} / \mathrm{s}$ for temperature range fro $\mathrm{m} 65$ to $85^{\circ} \mathrm{C}$ (Table 2). Moisture diffusivity of mango foam mats increased with increase in drying air temperature. Moisture diffusivity was maximum for $3 \%$ egg white at $65^{\circ} \mathrm{C}$ and $7 \%$ at $75^{\circ} \mathrm{C}$ along with highest $R^{2}$ values.

\subsection{Model Fitting}

The mo isture ratio data of foam mat dry ing of mango pulp at different temperatures using different concentrations of egg white were fitted into thin layer drying models (Table 1). The coefficient of correlation and results of statistical analysis are listed in Table 3. Four criteria for adequacy of the model fit, namely, coefficient of determination $\left(R^{2}\right)$, reduced Chi square $\left(\chi^{2}\right)$, mean biased error (MBE) and root mean square error (RMSE) were used. The best model describing the thin layer drying characteristics of mango foam mat drying was chosen as the one with the highest $\mathrm{R}^{2}$ and lowest $\chi^{2}$, MBE and RMSE. All the models fitted gave $\mathrm{R}^{2}$ more than 0.9 , however out of these, Wang and Singh model was the best fitted with $\mathrm{R}^{2}$ more than 0.99 for 65 and $85^{\circ} \mathrm{C}$, whereas, Logarithmic and diffusion approach model were found suitable for $75^{\circ} \mathrm{C}$.

Table 2. Effect ive moisture diffusivity and its linear equation for foam mat drying of mango pulp

\begin{tabular}{cccccc}
\hline $\begin{array}{c}\mathrm{Te} \\
\mathrm{mp} \\
\left({ }^{\circ} \mathrm{C}\right.\end{array}$ & EW & Equation & $\begin{array}{c}\mathrm{k} 0 \\
\text { values }\end{array}$ & $\mathrm{D}_{\text {eff }}$ & $\mathrm{R}^{2}$ \\
\hline $\begin{array}{c}{ }^{6} 5 \\
65\end{array}$ & 0 & $\mathrm{y}=-0.0042 \mathrm{x}-0.1599$ & -0.0042 & $1.53 \mathrm{E}-08$ & 0.9064 \\
& 3 & $\mathrm{y}=-0.0046 \mathrm{x}-0.0158$ & -0.0046 & $1.68 \mathrm{E}-08$ & 0.9627 \\
& 5 & $\mathrm{y}=-0.0045 \mathrm{x}-0.0643$ & -0.0045 & $1.64 \mathrm{E}-08$ & 0.9533 \\
& 7 & $\mathrm{y}=-0.0045 \mathrm{x}-0.0358$ & -0.0045 & $1.64 \mathrm{E}-08$ & 0.9424 \\
& 9 & $\mathrm{y}=-0.0044 \mathrm{x}-0.1005$ & -0.0044 & $1.60 \mathrm{E}-08$ & 0.9203 \\
75 & 0 & $\mathrm{y}=-0.0052 \mathrm{x}-0.1938$ & -0.0052 & $1.90 \mathrm{E}-08$ & 0.9296 \\
& 3 & $\mathrm{y}=-0.0051 \mathrm{x}-0.2904$ & -0.0051 & $1.86 \mathrm{E}-08$ & 0.8679 \\
& 5 & $\mathrm{y}=-0.0050 \mathrm{x}-0.3003$ & -0.0050 & $1.82 \mathrm{E}-08$ & 0.8630 \\
& 7 & $\mathrm{y}=-0.0052 \mathrm{x}-0.1405$ & -0.0052 & $1.90 \mathrm{E}-08$ & 0.9468 \\
& 9 & $\mathrm{y}=-0.0055 \mathrm{x}-0.1613$ & -0.0055 & $2.01 \mathrm{E}-08$ & 0.9309 \\
85 & 0 & $\mathrm{y}=-0.0071 \mathrm{x}+0.0616$ & -0.0071 & $2.59 \mathrm{E}-08$ & 0.9601 \\
& 3 & $\mathrm{y}=-0.0068 \mathrm{x}-0.0455$ & -0.0068 & $2.48 \mathrm{E}-08$ & 0.9136 \\
& 5 & $\mathrm{y}=-0.0070 \mathrm{x}+0.0368$ & -0.0070 & $2.55 \mathrm{E}-08$ & 0.9522 \\
& 7 & $\mathrm{y}=-0.0069 \mathrm{x}-0.0395$ & -0.0069 & $2.52 \mathrm{E}-08$ & 0.9123 \\
& 9 & $\mathrm{y}=-0.0072 \mathrm{x}+0.0556$ & -0.0072 & $2.63 \mathrm{E}-08$ & 0.9551 \\
\hline
\end{tabular}

\subsection{Total Carotene}

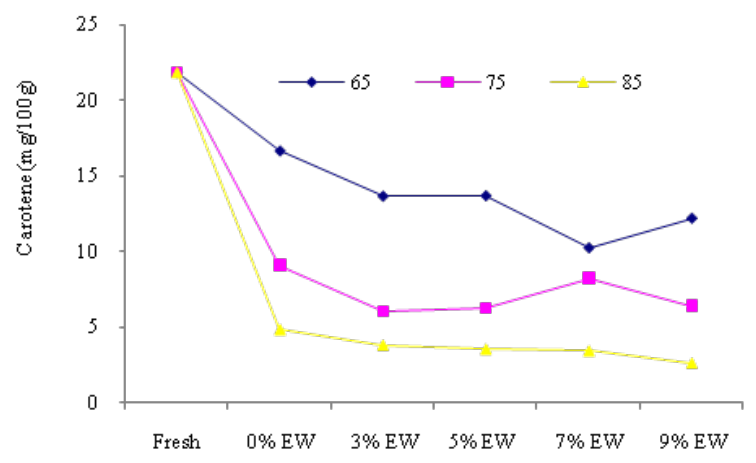

Figure 2. Changes in total carotenes with foam mat drying at 65,75 , and $85^{\circ} \mathrm{C}$ temperature

Mango is a major source of carotenes, which are known to be important since decades for various health benefits and role in disease prevention. Total carotene of fresh mango was found to be $21.84 \pm 3.88 \mathrm{mg} / 100 \mathrm{~g}$. Total carotene content of freshly prepared foam mat dried mango powder was in the range of $16.59-4.25 \mathrm{mg} / 100 \mathrm{~g}$. Foam mat drying at different temperature using different concentrations of egg white had significant effect on the carotene content (Figure 2). Decrease in carotene content was observed with increasing 
concentration of egg white and temperature. The loss of total dehydration was reported by Chen, Peng, \& Chen[19] in carotene could be attributed to its photosensitive nature, Taiwanese mango, Wen-ping, Zhi-jing, He, \& Min[20] in isomerization and epoxide forming nature of carotenoids[17, fruits of Lycjum barbarum and Lavelli, Zanoni, \& 18]. Decline was more pronounced in case of 75 and $85^{\circ} \mathrm{C}$ Zaniboni[21] in dehydrated carrots.

than at $65^{\circ} \mathrm{C}$. Decline in carotene content with drying and

Table 3. Statistical Quality Analysis of Fitted Thin Layer Drying Mathematical Modelsto Foam Mat Drying of Mango Pulp

\begin{tabular}{|c|c|c|c|c|c|c|c|c|c|c|}
\hline Model & Temp $\left({ }^{\circ} \mathrm{C}\right)$ & $\mathrm{EW} \%$ & $\mathrm{R}^{2}$ & $\chi^{2}$ & MBE & RMSE & \multicolumn{4}{|c|}{ Model Constants } \\
\hline \multirow[t]{15}{*}{ Newton } & 65 & 0 & 0.9819 & 0.0013 & 0.0037 & 0.0098 & $\mathrm{k}: 0.005$ & & & \\
\hline & & 3 & 0.9635 & 0.0025 & 0.0032 & 0.0132 & $\mathrm{k}: 0.005$ & & & \\
\hline & & 5 & 0.9836 & 0.0012 & 0.0029 & 0.0091 & $\mathrm{k}: 0.005$ & & & \\
\hline & & 7 & 0.9731 & 0.0020 & 0.0049 & 0.0120 & $\mathrm{k}: 0.005$ & & & \\
\hline & & 9 & 0.9720 & 0.0020 & 0.0039 & 0.0120 & $\mathrm{k}: 0.005$ & & & \\
\hline & 75 & 0 & 0.9617 & 0.0026 & 0.0023 & 0.0147 & $\mathrm{k}: 0.007$ & & & \\
\hline & & 3 & 0.9272 & 0.0051 & 0.0071 & 0.0204 & $\mathrm{k}: 0.008$ & & & \\
\hline & & 5 & 0.9201 & 0.0055 & 0.0072 & 0.0213 & $\mathrm{k}: 0.008$ & & & \\
\hline & & 7 & 0.9723 & 0.0019 & 0.0024 & 0.0127 & $\mathrm{k}: 0.006$ & & & \\
\hline & & 9 & 0.9705 & 0.0022 & 0.0047 & 0.0135 & $\mathrm{k}: 0.007$ & & & \\
\hline & 85 & 0 & 0.9700 & 0.0028 & 0.0079 & 0.0160 & $\mathrm{k}: 0.007$ & & & \\
\hline & & 3 & 0.9689 & 0.0028 & 0.0074 & 0.0159 & $\mathrm{k}: 0.007$ & & & \\
\hline & & 5 & 0.9839 & 0.0014 & 0.0043 & 0.0112 & $\mathrm{k}: 0.007$ & & & \\
\hline & & 7 & 0.9734 & 0.0023 & 0.0058 & 0.0145 & $\mathrm{k}: 0.008$ & & & \\
\hline & & 9 & 0.9806 & 0.0018 & 0.0050 & 0.0126 & $\mathrm{k}: 0.007$ & & & \\
\hline \multirow[t]{15}{*}{ Hederson and Pebis } & 65 & 0 & 0.9859 & 0.0022 & 0.0036 & 0.0120 & $\mathrm{k}: 0.005$ & $\mathrm{a}: 0.976$ & & \\
\hline & & 3 & 0.9822 & 0.0026 & 0.0056 & 0.0130 & $\mathrm{k}: 0.005$ & $\mathrm{a}: 1.003$ & & \\
\hline & & 5 & 0.9918 & 0.0013 & 0.0022 & 0.0091 & $\mathrm{k}: 0.005$ & $\mathrm{a}: 1.006$ & & \\
\hline & & 7 & 0.9873 & 0.0021 & 0.0014 & 0.0117 & $\mathrm{k}: 0.005$ & $\mathrm{a}: 1.027$ & & \\
\hline & & 9 & 0.9859 & 0.0022 & 0.0036 & 0.0120 & $\mathrm{k}: 0.005$ & $\mathrm{a}: 1.003$ & & \\
\hline & 75 & 0 & 0.9833 & 0.0025 & 0.0069 & 0.0137 & $\mathrm{k}: 0.007$ & $\mathrm{a}: 0.953$ & & \\
\hline & & 3 & 0.9671 & 0.0050 & 0.0115 & 0.0193 & $\mathrm{k}: 0.007$ & $\mathrm{a}: 0.938$ & & \\
\hline & & 5 & 0.9641 & 0.0054 & 0.0119 & 0.0200 & $\mathrm{k}: 0.007$ & $\mathrm{a}: 0.933$ & & \\
\hline & & 7 & 0.9868 & 0.0020 & 0.0052 & 0.0123 & $\mathrm{k}: 0.006$ & $\mathrm{a}: 0.975$ & & \\
\hline & & 9 & 0.9855 & 0.0024 & 0.0065 & 0.0134 & $\mathrm{k}: 0.007$ & $\mathrm{a}: 0.982$ & & \\
\hline & 85 & 0 & 0.9886 & 0.0024 & -0.0009 & 0.0139 & $\mathrm{k}: 0.007$ & $\mathrm{a}: 1.061$ & & \\
\hline & & 3 & 0.9852 & 0.0030 & 0.0037 & 0.0154 & $\mathrm{k}: 0.007$ & $\mathrm{a}: 1.061$ & & \\
\hline & & 5 & 0.9928 & 0.0014 & 0.0003 & 0.0106 & $\mathrm{k}: 0.007$ & $\mathrm{a}: 1.029$ & & \\
\hline & & 7 & 0.9870 & 0.0026 & 0.0035 & 0.0143 & $\mathrm{k}: 0.008$ & $\mathrm{a}: 1.019$ & & \\
\hline & & 9 & 0.9919 & 0.0016 & -0.0006 & 0.0115 & $\mathrm{k}: 0.007$ & $\mathrm{a}: 1.041$ & & \\
\hline \multirow[t]{15}{*}{ Logrithmic } & 65 & 0 & 0.9917 & 0.0015 & 0.0000 & 0.0093 & $\mathrm{k}: 0.009$ & $\mathrm{a}: 0.857$ & c: 0.173 & \\
\hline & & 3 & 0.9943 & 0.0009 & 0.0000 & 0.0074 & $\mathrm{k}: 0.005$ & $\mathrm{a}: 0.995$ & c: 0.035 & \\
\hline & & 5 & 0.9939 & 0.0010 & 0.0000 & 0.0078 & $\mathrm{k}: 0.006$ & $\mathrm{a}: 0.926$ & c: 0.102 & \\
\hline & & 7 & 0.9881 & 0.0021 & 0.0000 & 0.0113 & $\mathrm{k}: 0.006$ & $\mathrm{a}: 0.971$ & c: 0.070 & \\
\hline & & 9 & 0.9905 & 0.0017 & 0.0000 & 0.0099 & $\mathrm{k}: 0.007$ & $\mathrm{a}: 0.908$ & c: 0.128 & \\
\hline & 75 & 0 & 0.9988 & 0.0002 & 0.0000 & 0.0036 & $\mathrm{k}: 0.011$ & $\mathrm{a}: 0.835$ & c: 0.177 & \\
\hline & & 3 & 0.9973 & 0.0005 & 0.0000 & 0.0056 & $\mathrm{k}: 0.014$ & $\mathrm{a}: 0.830$ & c: 0.190 & \\
\hline & & 5 & 0.9973 & 0.0005 & 0.0000 & 0.0056 & $\mathrm{k}: 0.015$ & $\mathrm{a}: 0.825$ & c: 0.195 & \\
\hline & & 7 & 0.9973 & 0.0005 & 0.0000 & 0.0055 & $\mathrm{k}: 0.010$ & $\mathrm{a}: 0.854$ & c: 0.170 & \\
\hline & & 9 & 0.9963 & 0.0007 & 0.0000 & 0.0068 & $\mathrm{k}: 0.011$ & $\mathrm{a}: 0.876$ & c: 0.155 & \\
\hline & 85 & 0 & 0.9888 & 0.0027 & 0.0000 & 0.0138 & k:0.007 & $\mathrm{a}: 1.094$ & c:-0.039 & \\
\hline & & 3 & 0.9878 & 0.0028 & 0.0000 & 0.0140 & $\mathrm{k}: 0.010$ & a:0.961 & c: 0.091 & \\
\hline & & 5 & 0.9928 & 0.0016 & 0.0000 & 0.0106 & $\mathrm{k}: 0.007$ & $\mathrm{a}: 1.020$ & c: 0.012 & \\
\hline & & 7 & 0.9893 & 0.0024 & 0.0000 & 0.0130 & $\mathrm{k}: 0.010$ & $\mathrm{a}: 0.953$ & c: 0.086 & \\
\hline & & 9 & 0.9920 & 0.0019 & 0.0000 & 0.0114 & $\mathrm{k}: 0.007$ & $\mathrm{a}: 1.061$ & c:- -0.025 & \\
\hline \multirow[t]{8}{*}{ Two term } & 65 & 0 & 0.9922 & 0.0015 & 0.0013 & 0.0090 & $\mathrm{k}: 1.000$ & $\mathrm{a}: 0.051$ & c:0.949 & b:0.005 \\
\hline & & 3 & 0.9830 & 0.0030 & 0.0046 & 0.0127 & $\mathrm{k}: 1.000$ & a:-0.048 & c:1.048 & b:0.005 \\
\hline & & 5 & 0.9918 & 0.0016 & 0.0024 & 0.0091 & $\mathrm{k}: 1.000$ & a:-0.012 & c:1.012 & b:0.005 \\
\hline & & 7 & 0.9881 & 0.0024 & 0.0023 & 0.0113 & $\mathrm{k}: 1.000$ & $\mathrm{a}:-0.055$ & c:1.055 & b:0.005 \\
\hline & & 9 & 0.9859 & 0.0027 & 0.0037 & 0.0120 & $\mathrm{k}: 1.000$ & $\mathrm{a}:-0.005$ & c:1.005 & b:0.005 \\
\hline & 75 & 0 & 0.9875 & 0.0024 & 0.0040 & 0.0119 & $\mathrm{k}: 1.000$ & a: 0.119 & $\mathrm{c}: 0.881$ & b:0.006 \\
\hline & & 3 & 0.9753 & 0.0048 & 0.0060 & 0.0167 & $\mathrm{k}: 1.000$ & a: 0.173 & c:0.827 & b:0.006 \\
\hline & & 5 & 0.9737 & 0.0051 & 0.0059 & 0.0171 & $\mathrm{k}: 1.000$ & a: 0.186 & c:0.814 & b:0.006 \\
\hline
\end{tabular}


Table 3. Continued

\begin{tabular}{|c|c|c|c|c|c|c|c|c|c|c|}
\hline Model & Temp $\left({ }^{\circ} \mathrm{C}\right)$ & $\mathrm{EW} \%$ & $\mathrm{R}^{2}$ & $\chi^{2}$ & MBE & RMSE & \multicolumn{4}{|c|}{ Model Constants } \\
\hline & & 7 & 0.9880 & 0.0024 & 0.0039 & 0.0118 & $\mathrm{k}: 1.000$ & a: 0.062 & c:0.938 & b:0.006 \\
\hline & & 9 & 0.9861 & 0.0030 & 0.0053 & 0.0131 & $\mathrm{k}: 1.000$ & a: 0.048 & c:0.952 & b:0.007 \\
\hline & 85 & 0 & 0.9946 & 0.0015 & 0.0022 & 0.0096 & $\mathrm{k}: 1.000$ & $\mathrm{a}:-0.167$ & c:1.167 & b:0.008 \\
\hline & & 3 & 0.9946 & 0.0015 & 0.0022 & 0.0096 & $\mathrm{k}: 1.000$ & $\mathrm{a}:-0.167$ & c:1.167 & b:0.008 \\
\hline & & 5 & 0.9943 & 0.0015 & 0.0018 & 0.0095 & $\mathrm{k}: 1.000$ & $\mathrm{a}:-0.078$ & c:1.078 & b:0.007 \\
\hline & & 7 & 0.9876 & 0.0032 & 0.0049 & 0.0140 & $\mathrm{k}: 1.000$ & $\mathrm{a}:-0.055$ & c:1.055 & b:0.008 \\
\hline & & 9 & 0.9946 & 0.0015 & 0.0014 & 0.0094 & $\mathrm{k}: 1.000$ & $\mathrm{a}:-0.110$ & c: 1.110 & b:0.008 \\
\hline \multirow[t]{7}{*}{ Two term expot ential } & 65 & 0 & 0.9907 & 0.0015 & 0.0037 & 0.0098 & $\mathrm{k}: 0.012$ & $\mathrm{a}: 0.313$ & & \\
\hline & & 3 & 0.9886 & 0.0017 & 0.0034 & 0.0104 & $\mathrm{k}: 1.595$ & $\mathrm{a}: 0.003$ & & \\
\hline & & 5 & 0.9917 & 0.0013 & 0.0028 & 0.0092 & k:0.929 & $\mathrm{a}: 0.005$ & & \\
\hline & & 7 & 0.9863 & 0.0022 & 0.0048 & 0.0121 & $\mathrm{k}: 1.759$ & $\mathrm{a}: 0.003$ & & \\
\hline & & 9 & 0.9858 & 0.0022 & 0.0038 & 0.0120 & $\mathrm{k}: 0.948$ & $\mathrm{a}: 0.005$ & & \\
\hline & 75 & 0 & 0.9925 & 0.0011 & 0.0022 & 0.0092 & $\mathrm{k}: 0.020$ & $\mathrm{a}: 0.261$ & & \\
\hline & & 3 & 0.9812 & 0.0029 & 0.0046 & 0.0146 & $\mathrm{k}: 0.023$ & $\mathrm{a}: 0.262$ & & \\
\hline \multirow[t]{8}{*}{ Two term expotential } & 75 & 5 & 0.9792 & 0.0031 & 0.0046 & 0.0153 & $\mathrm{k}: 0.023$ & $\mathrm{a}: 0.258$ & & \\
\hline & & 7 & 0.9933 & 0.0010 & 0.0030 & 0.0088 & $\mathrm{k}: 0.016$ & $\mathrm{a}: 0.294$ & & \\
\hline & & 9 & 0.9915 & 0.0014 & 0.0041 & 0.0102 & $\mathrm{k}: 0.016$ & $\mathrm{a}: 0.320$ & & \\
\hline & 85 & 0 & 0.9847 & 0.0032 & 0.0078 & 0.0161 & $\mathrm{k}: 4.703$ & $\mathrm{a}: 0.001$ & & \\
\hline & & 3 & 0.9842 & 0.0032 & 0.0070 & 0.0159 & $\mathrm{k}: 3.155$ & $\mathrm{a}: 0.002$ & & \\
\hline & & 5 & 0.9918 & 0.0016 & 0.0042 & 0.0113 & $\mathrm{k}: 2.964$ & $\mathrm{a}: 0.002$ & & \\
\hline & & 7 & 0.9865 & 0.0027 & 0.0057 & 0.0146 & $\mathrm{k}: 2.474$ & $\mathrm{a}: 0.003$ & & \\
\hline & & 9 & 0.9901 & 0.0020 & 0.0046 & 0.0127 & $\mathrm{k}: 3.610$ & $\mathrm{a}: 0.002$ & & \\
\hline \multirow[t]{15}{*}{ Wang and Singh } & 65 & 0 & 0.9950 & 0.0008 & 0.0038 & 0.0072 & $\mathrm{a}:-0.005$ & b:0.000 & & \\
\hline & & 3 & 0.9978 & 0.0003 & 0.0000 & 0.0046 & $\mathrm{a}:-0.004$ & b:0.000 & & \\
\hline & & 5 & 0.9976 & 0.0004 & 0.0023 & 0.0049 & a:-0.005 & b:0.000 & & \\
\hline & & 7 & 0.9925 & 0.0012 & 0.0054 & 0.0090 & $\mathrm{a}:-0.004$ & b:0.000 & & \\
\hline & & 9 & 0.9961 & 0.0006 & 0.0030 & 0.0063 & $\mathrm{a}:-0.005$ & b:0.000 & & \\
\hline & 75 & 0 & 0.9936 & 0.0010 & -0.0052 & 0.0085 & $\mathrm{a}:-0.007$ & b:0.000 & & \\
\hline & & 3 & 0.9836 & 0.0025 & -0.0077 & 0.0137 & $\mathrm{a}:-0.007$ & b:0.000 & & \\
\hline & & 5 & 0.9815 & 0.0028 & -0.0083 & 0.0144 & $\mathrm{a}:-0.007$ & b:0.000 & & \\
\hline & & 7 & 0.9960 & 0.0006 & -0.0016 & 0.0068 & $\mathrm{a}:-0.006$ & b:0.000 & & \\
\hline & & 9 & 0.9955 & 0.0007 & -0.0012 & 0.0074 & a:-0.007 & b:0.000 & & \\
\hline & 85 & 0 & 0.9906 & 0.0020 & 0.0091 & 0.0126 & a:- -0.006 & b:0.000 & & \\
\hline & & 3 & 0.9927 & 0.0015 & 0.0063 & 0.0108 & $\mathrm{a}:-0.007$ & b:0.000 & & \\
\hline & & 5 & 0.9963 & 0.0007 & 0.0043 & 0.0076 & a:-0.006 & b:0.000 & & \\
\hline & & 7 & 0.9950 & 0.0010 & 0.0042 & 0.0089 & $\mathrm{a}:-0.007$ & b:0.000 & & \\
\hline & & 9 & 0.9951 & 0.0010 & 0.0055 & 0.0089 & $\mathrm{a}:-0.006$ & b:0.000 & & \\
\hline \multirow[t]{15}{*}{ Diffusion approach } & 65 & 0 & 0.9926 & 0.0013 & 0.0014 & 0.0088 & $\mathrm{k}: 0.006$ & a: 0.997 & $\mathrm{c}:-1$ & \\
\hline & & 3 & 0.9971 & 0.0005 & 0.0020 & 0.0053 & $\mathrm{k}: 0.038$ & $\mathrm{a}:-0.074$ & c: 0 & 35 \\
\hline & & 5 & 0.9918 & 0.0014 & 0.0024 & 0.0091 & $\mathrm{k}: 0.119$ & $\mathrm{a}:-0.012$ & c: 0 & 42 \\
\hline & & 7 & 0.9883 & 0.0021 & 0.0024 & 0.0112 & $\mathrm{k}: 0.046$ & $\mathrm{a}:-0.075$ & c: 0 & 12 \\
\hline & & 9 & 0.9859 & 0.0024 & 0.0037 & 0.0120 & $\mathrm{k}: 0.102$ & $\mathrm{a}:-0.006$ & c: 0 & 51 \\
\hline & 75 & 0 & 0.9993 & 0.0001 & 0.0007 & 0.0028 & $\mathrm{k}: 0.009$ & a: 0.958 & c:- -0 & 186 \\
\hline & & 3 & 0.9978 & 0.0004 & 0.0016 & 0.0050 & $\mathrm{k}: 0.012$ & a: 0.928 & $\mathrm{c}:-0$ & 80 \\
\hline & & 5 & 0.9977 & 0.0004 & 0.0016 & 0.0051 & $\mathrm{k}: 0.012$ & a: 0.915 & $c:-0$ & 33 \\
\hline & & 7 & 0.9978 & 0.0004 & 0.0020 & 0.0050 & $\mathrm{k}: 0.008$ & a: 0.986 & $\mathrm{c}:-0$ & 67 \\
\hline & & 9 & 0.9971 & 0.0005 & 0.0026 & 0.0060 & $\mathrm{k}: 0.008$ & a: 0.992 & $c:-1$ & 15 \\
\hline & 85 & 0 & 0.9948 & 0.0013 & 0.0026 & 0.0094 & $\mathrm{k}: 0.071$ & $\mathrm{a}:-0.195$ & c: 0 & 14 \\
\hline & & 3 & 0.9870 & 0.0030 & 0.0060 & 0.0145 & $\mathrm{k}: 0.300$ & $\mathrm{a}:-0.091$ & c: 0 & 28 \\
\hline & & 5 & 0.9944 & 0.0013 & 0.0020 & 0.0094 & $\mathrm{k}: 0.061$ & a:- -0.098 & c: 0 & 26 \\
\hline & & 7 & 0.9876 & 0.0028 & 0.0049 & 0.0140 & $\mathrm{k}: 0.204$ & $\mathrm{a}:-0.055$ & c: 0 & 40 \\
\hline & & 9 & 0.9949 & 0.0012 & 0.0019 & 0.0091 & $\mathrm{k}: 0.052$ & $\mathrm{a}:-0.150$ & c: 0 & 54 \\
\hline
\end{tabular}




\section{Conclusions}

From the present study on foam mat drying of mango pulp, it can be concluded that increase in drying temperature decreased drying time. Foaming with different

egg white concentrations had significant effect on drying up to the level of $3 \%$ as evident from mo isture ratio curves and diffusivity data. Further increase in egg white concentration either had similar or negative impact on drying. Foam mat drying data followed Wang and Singh model for 65 and $85^{\circ} \mathrm{C}$ and Logarithmic and diffusion approach for $75^{\circ} \mathrm{C}$. Total carotene content was highest in case of samples dried at $65^{\circ} \mathrm{C}$. In nutshell, it can be recommended that foam mat dry ing of mango pulp can be carried out using 3\% egg white as foaming agent and $65^{\circ} \mathrm{C}$ air drying temperature with the retention of nutritional quality.

\section{ACKNOWLEDGEMENTS}

The authors wish to express sincere thanks to Department of Science and Technology (DST), New Delhi for providing financial as sistance to carry out this work.

\section{REFERENCES}

[1] Hymavathi TV, and Khader V, Carotene, ascorbic acid and sugar content of vacuum dehydrated ripe mango powders stored in flexible packaging material. Journal of Food Composition and Analysis, 18, 181-192, 2005.

[2] Pandit SS, Chidley HG, Kulkarni RS, Pujari KH, Giri AP, and Gupta VS, Cultivar relationship in mango based on fruit volatile profiles. Food Chemistry, 114, 363-372, 2009.

[3] Mitra SK and Baldwin EA, Mango. Postharvest physiology and storage of tropical and subtropical fruits. pp. 85-122. Wallingford, UK: CAB International, 1997.

[4] Hassan M, and Ahmed J, Physico-chemical and sensory characteristics of man go-milk beverage. Indian Food Packer, 52, 32-36, 1998.

[5] Baldry J, Breay, GR, Cay gill TC, Cook RD, Ferber CEM and Lalitha K, Alternative methods of processing mangoes. Indian Food Packer, 30, 50-62, 1976.

[6] Ammu K and Krishnappa K G, Freeze drying of mango pulp. J. Food Tech., 28, 339, 1976.

[7] Nanjundaswamy AM, Studies on preparation of dehydrated products from tropical fruits and their pulps. Ph. D. Thesis, University of Mysore, Karnataka, India, 1984.

[8] Ratti C and Kudra T, Drying of foamed materials opportunities and challenges. In proceeding 11th polish Drying symposium. Sept, pp. 13-16. CD-ROM. Poznar, Poland, 2005.

[9] Kadam D M and Balasubramanian S, Foam mat drying of tomato juice. Journal of food processing and preservation, 35(4), 488-495, 2011.
[10] Morgan AI, Graham RP, Ginnette LF and Williams GS, Recent developments in foam mat dry ing. Food technology, 15, 37-39, 1961 .

[11] Hart MR, Graham RP, Ginnette LF and Morgan AI, Foams for foam-mat drying. Food Technology, 17, 1302-1304, 1963.

[12] Goyal RK, Kingsly ARP, Manikantan MR, and Ilyas SM, Mathematical Modelling of Thin Layer Drying Kinetics of Plum in a Tunnel Dryer. Journal of Food Engineering, 79, 176-180, 2007.

[13] Doymaz I, Thin layer drying behaviour of mint leaves. Journal of Food Engineering, 74, 370-375, 2006.

[14] Crank J, Mathematics of diffusion., Oxford, UK: Clarendon Press. 1975.

[15] Erenturk S, Gulaboglu MS and Gultekin S, The thin layer dry ing characteristics of rosehip. Biosystems Engineering, 89 (2), 159-166, 2004.

[16] Rangana S, Hand book of analysis and quality control for fruits and vegetable products. (2nd ed.). pp. 1112. New Delhi: Tata McGraw- Hill publication Co. Ltd, 1995.

[17] Eskin NAM, Plant Pigment Flavours and Textures. The Chemistry and Biochemistry of Selected Compounds.pp. 219 London: Academic Press, 1979.

[18] Mir MA, and Nath N, Storage chan ges in fortified man go bars. Journal of Food Science and Technology, 30, 279-287, 1993.

[19] Chen B H, Peng, H Y and Chen H E, Changes of carotenoids, colour and vitamins A contents during processing of carrot juice. J A gri. Food Chem. 43, 1912-1918, 1995.

[20] Wen-Ping M, Zhi-Jing N, He L and Min C, Changes of the Main Carotenoid Pigment Contents during the drying processes of the different harvest stage fruits of Lycium barbarum L. A gricultural Sciences in China, 7, 363-369, 2008.

[21] Lavelli V, Zanoni B, and Zaniboni A, Effect of water activity on carotenoid degradation in dehydrated carrots. Food Chemistry, 104, 1705-1711, 2007.

[22] Mujumdar A S, Handbook of industrial drying. New York: Marcel Dekker, 1987.

[23] Henderson SM and Pabis S, Grain drying theory I. Temperature effect on drying coefficient. Journal of Agricultural En gin eering Research, 6 (3), 169-174, 1961.

[24] Chhinnman MS, Evaluation of selected mathematical models for describing thin layer drying of in-shell pecans. Transactions of the ASAE, 27, 610-615, 1984.

[25] Yagcioglu A, Degirmencioglu A and Cagatay F, Drying characteristic of laurel leaves under different conditions, in: A. Bascetincelik (Eds.), Proceedings of the 7th international congress on agricultural mechanization and energy, Faculty of Agriculture, Cukurova University, Adana, Turkey, pp. 565-569, 1999.

[26] Henderson S M, Progress in developing the thin layer drying equation. Transactions of the ASAE, 17, 1167-1172, 1974.

[27] Madamba PS, Driscoll RH and Buckle KA, The thin layer drying characteristic of garlic slices. Journal of Food Engineering, 29, 75-97, 1996. 
[28] Sharaf-Eldeen Y I, Blaisdell J L and Hamdy M Y, A model for ear corn dry ing. Transaction of the ASAE, 23, 1261-1271, 1980.

[29] Wang C Y and Singh R P, Use of variable equilibrium moisture content in modelling rice dry ing. Transaction of the ASAE, 11, 668-672, 1978.

[30] Yaldiz O and Ertekin C, Thin layer solar drying of some vegetables. Drying Technology, 19, 583-596, 2001. 